\title{
TITLE:
}

\section{Coherent swing instability of power systems and cascading failures}

$\operatorname{AUTHOR}(\mathrm{S})$ :

Susuki, Yoshihiko; Mezi, Igor; Hikihara, Takashi

\section{CITATION:}

Susuki, Yoshihiko ...[et al]. Coherent swing instability of power systems and cascading failures. Proceedings of the 2010 IEEE Power \& Energy Society General Meeting (PESGM2010) 2010:944.

\section{ISSUE DATE:}

2010-07

URL:

http://hdl.handle.net/2433/123309

\section{RIGHT:}

(02010 IEEE. Personal use of this material is permitted. However, permission to reprint/republish this material for advertising or promotional purposes or for creating new collective works for resale or redistribution to servers or lists, or to reuse any copyrighted component of this work in other works must be obtained from the IEEE. 


\section{Coherent Swing Instability of Power Systems and Cascading Failures}

\author{
Yoshihiko Susuki \\ Department of Mechanical Engineering \\ University of California, Santa Barbara \\ CA 93106-5370, United States \\ and \\ Department of Electrical Engineering \\ Kyoto University \\ Nishikyo, Kyoto 615-8510, Japan \\ Email: susuki@ieee.org
}

\author{
Igor Mezić \\ Department of Mechanical Engineering \\ University of California, Santa Barbara \\ CA 93106-5370, United States \\ Email: mezic@engineering.ucsb.edu
}

\author{
Takashi Hikihara \\ Department of Electrical Engineering \\ Kyoto University \\ Nishikyo, Kyoto 615-8510, Japan \\ Email: hikihara@kuee.kyoto-u.ac.jp
}

\begin{abstract}
We describe short-term swing dynamics leading to cascading failure in an interconnected power system. The notion of Coherent Swing Instability (CSI) was recently proposed in which most of synchronous machines in a power system coherently lose synchronism with the rest of the system after being subjected to a local and finite disturbance. CSI is an escape phenomenon from resonance in the nonlinear mode that governs the collective motion of machines. For a network of weakly interconnected power systems, we show that CSI happens for all of the systems in a cascade manner and gives the mechanism of cascading failure in the network, that is, the sequence of escape phenomena that can occur in a class of high-dimensional dynamical systems.
\end{abstract}

\section{INTRODUCTION}

Coupled swing dynamics in a population of synchronous machines are of vital importance for power system stability. The so-called transient stability analysis is associated with the ability of power system to maintain synchronism when subjected to a large disturbance [1], [2]. Loss of transient stability is recognized as one cause of large blackouts such as the September 2003 blackout in Italy [3]. Transient stability is mainly governed by electromechanical or rotor angle oscillations of synchronous machines in short-term regime (0 to 10 seconds [1]) and is mathematically investigated by the so-called nonlinear swing equations [1], [2]. Analysis of the swing equations (see e.g. [4]-[8]), which especially deals with global structures of phase space far from equilibria, is hence needed for prevention of not only transient instability but also large blackouts.

In [9]-[13] we have developed a theory of short-term swing instability of multi-machine power systems, which we term the Coherent Swing Instability (CSI), based on the notion of instability occurring for general oscillatory systems described in [14]-[17]. CSI is an undesirable and emergent phenomenon of synchronous machines in a power system, in which machines in a subset of the system coherently lose synchronism with the rest of the system after being subjected to a finite and local disturbance. ${ }^{1}$ This phenomenon gives the dynamical mechanism that explains how local plant mode oscillation, inter-area mode instability, and multi-swing instability interact to destabilize a power system. In [9], [11], [12], we presented the phenomenology and mechanism of CSI for simple and practical models of power systems. CSI occurs in a class of dynamical systems dominated by inertia in which one nonlinear mode is weak compared with many linear oscillatory modes. The nonlinear mode governs the collective motion of synchronous machines in a power system and is invisible in normal model representations, but it can be extracted using analytical tools of Center-Of-Angle (COA) [19] and slowcoherency [20], or computations of proper orthonormal modes [10] and Koopman modes [21]. With this modal perspective, we dynamically state that CSI is an escape phenomenon (see [22], [23]) from resonance (see [24]) in the nonlinear mode. The nonlinear mode features a special phase space structure of resonance: resonance of all orders [17]. The resonant surface is a codimension-one subspace in the full phase space. Then the escape of trajectory from the resonant surface corresponds to CSI. A stable region of phase space in which any trajectory does not escape is evaluated using the ergodic partition theorem [13].

In this paper, on the basis of CSI, we describe shortterm swing dynamics leading to cascading failure in an interconnected power system. Cascading failure is a sequence of dependent failures of individual components that successively weakens the power system [25]. Understanding the physics of cascading failures is of basic importance for design and control of power systems. There is a large amount of past and current work on the physics of cascading failures as reviewed in [11], [25]. In this paper, after reviewing the theory of CSI for a

\footnotetext{
${ }^{1}$ This notion of instability is somewhat broader than the standard definition in dynamical systems [18], in the sense that it does not happen upon an infinitesimally small perturbation around an equilibrium of the system. However, it encompasses the situation when the system escapes a predefined set around the equilibrium. Thus the notion of instability that we use here is non-local.
} 


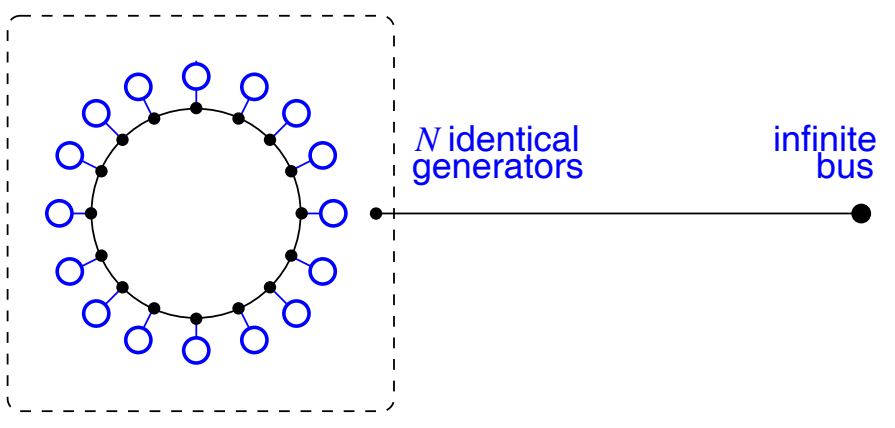

Fig. 1. A model of loop power system. Small blue circles represent synchronous generators. They operate in the AC loop network and are connected to the infinite bus.

loop power system, we numerically present a phenomenon of short-term swing dynamics leading to cascading failure of a network of weakly interconnected power systems. The network consists of multiple loop power systems, each of which is used in the review part, coupled via weak transmission lines in series. We study nonlinear swing equations for analysis of short-term swing dynamics in the network. Numerical simulation shows that CSI happens for all the loop systems in a cascade manner. CSI in every system corresponds to its transient instability and hence to a sub-system failure. Then we investigate the cascading failure by projecting the fullsystem dynamics onto phase planes of COA variables, thereby describing the sequence of escape phenomena underlying in the cascading failure. These escapes result from dynamic interactions between loop systems. Thus we suggest that CSI could be a part of the dynamical mechanism of cascading failures in large-scale power systems.

\section{A Review of Coherent Swing Instability}

In this section, we review the theory of Coherent Swing Instability (CSI) using a loop power system. After introducing the nonlinear swing equations (Sec. II-B), we present the phenomenon and mechanism of CSI (Secs. II-C and II-D, respectively). The contents of this section are submitted to an archival journal [11].

\section{A. A Model of Loop Power System}

Consider the simple loop power system shown in Fig. 1. The system consists of $N$ identical generators, encompassed by the dotted box, which operate in the AC loop network and are connected to the infinite bus. We make the following assumptions. (i) The generators are small, and the transmission lines joining the infinite bus and a generator are much longer than those joining two generators in the loop network. Thus the magnitude of interaction of an individual generator with the infinite bus is smaller than the magnitude of interaction between any two generators. (ii) The lengths of transmission lines between the infinite bus and individual generators are identical. (iii) The lengths of transmission lines between generators are identical. (iv) The power system is loss-less. (v) The transformer inductance and synchronous reactance of each generator are negligible. These assumptions enable us to derive a simple mathematical model for analysis of CSI and can be relaxed substantially, while still observing the same phenomenon: see [9], [11], [12].

\section{B. The Swing Equations}

We introduce the equations of motion for the loop power system. We denote by $\delta_{i}$ the angular position of rotor with respect to the infinite bus of generators labeled with integer values $i=1, \ldots, N$. The non-dimensional deviation of rotor speed in generator $i$ relative to the system angular frequency (normally $2 \pi \times 50 \mathrm{rad} / \mathrm{s}$ or $2 \pi \times 60 \mathrm{rad} / \mathrm{s}$ ) is denoted by $\omega_{i}$. The short-term dynamics of generator $i$ are represented by the following nonlinear swing equations:

$$
\left.\begin{array}{rl}
\frac{\mathrm{d} \delta_{i}}{\mathrm{~d} t}= & \omega_{i}, \\
\frac{\mathrm{d} \omega_{i}}{\mathrm{~d} t}= & p_{\mathrm{m}}-b \sin \delta_{i}-b_{\text {int }}\left\{\sin \left(\delta_{i}-\delta_{i-1}\right)\right. \\
& \left.+\sin \left(\delta_{i}-\delta_{i+1}\right)\right\} .
\end{array}\right\}
$$

The loop connection of the generators induces the following conditions:

$$
\delta_{0}(t)=\delta_{N}(t), \quad \delta_{N+1}(t)=\delta_{1}(t) .
$$

The parameters $p_{\mathrm{m}}, b$, and $b_{\mathrm{int}}$ are constant in time and are in per unit system. The constant $p_{\mathrm{m}}$ is the mechanical input power to a generator, $b$ is the critical transmission power between the infinite bus and a generator, and $b_{\text {int }}$ is the critical transmission power between any two generators.

Now we simplify (1) in order to construct a mathematical model of the phenomenon of interest, where the differences between individual angular positions stay small for all time. There are trajectories of (1) that stay on a two-dimensional invariant manifold $\mathcal{I}$ in $2 N$-dimensional phase space of the system, defined by the $2(N-1)$ constraints $\delta_{i}=\delta_{i+1}$ and $\omega_{i}=\omega_{i+1}$ for $i=1, \ldots, N-1$. These are trajectories with the common initial conditions $\delta_{i}(0)$ and $\omega_{i}(0)$ for all the generators. We investigate the dynamics of (1) close to this invariant manifold. This is equivalent to the condition that the differences $\delta_{i}(t)-\delta_{i+1}(t)$ and $\omega_{i}(t)-\omega_{i+1}(t)$ for $i=1, \ldots, N-1$ are sufficiently small. This allows us to use the first-order approximation of the sinusoidal coupling term $b_{\text {int }}\left\{\sin \left(\delta_{i}-\delta_{i-1}\right)+\sin \left(\delta_{i}-\delta_{i+1}\right)\right\}$ and to derive the following system:

$$
\left.\begin{array}{rl}
\frac{\mathrm{d} \delta_{i}}{\mathrm{~d} t}= & \omega_{i}, \\
\frac{\mathrm{d} \omega_{i}}{\mathrm{~d} t}= & p_{\mathrm{m}}-b \sin \delta_{i}-b_{\mathrm{int}}\left\{\left(\delta_{i}-\delta_{i-1}\right)\right. \\
& \left.+\left(\delta_{i}-\delta_{i+1}\right)\right\} .
\end{array}\right\}
$$

The system (2) is an $N$ degree-of-freedom Hamiltonian system with the following Hamiltonian function $\mathcal{H}\left(\delta_{1}, \ldots, \delta_{N}, \omega_{1}, \ldots, \omega_{N}\right):$

$$
\mathcal{H}=\sum_{i=1}^{N} \frac{\omega_{i}^{2}}{2}-\sum_{i=1}^{N}\left\{p_{\mathrm{m}} \delta_{i}+b \cos \delta_{i}-\frac{b_{\text {int }}}{2}\left(\delta_{i-1}-\delta_{i}\right)^{2}\right\} .
$$


In (2) the linear term is an approximation of the sinusoidal characteristics in power transfer between nearest generators. This approximation is physically valid under the above assumption (i) that the length of transmission lines between nearest generators is much shorter than that between the infinite bus and an individual generator. That is, we have $b_{\text {int }} \gg b$, and the strong local coupling keeps the connected generators in the loop network close in angular position and speed deviation. Furthermore, the term $p_{\mathrm{m}}-b \sin \delta_{i}$ represents the nonlinear power-angle characteristics of each generator connected to the infinite bus and suggests an inherent possibility of loss of synchronism with the infinite bus. This nonlinearity is weak for the above assumption (i) of small generators, that is, $p_{\mathrm{m}}, b \ll b_{\text {int }}$. Thus, we investigate coupled swing dynamics in the power system with weak inter-system connection and strong inter-machine one.

\section{A Phenomenology}

In this section, we present numerical simulations of coupled swing dynamics represented by (2). The parameter settings for (2) used throughout most of this paper are

$$
p_{\mathrm{m}}=0.95, b=1, b_{\text {int }}=100, N=20 .
$$

The values of $p_{\mathrm{m}} / b_{\text {int }}=0.0095$ and $b / b_{\text {int }}=0.01$ are chosen to satisfy the above physical assumptions that imply strong linear coupling of generators and weak local nonlinearity governing the dynamics of each individual generator. All numerical integrations of the Hamiltonian system (2) were performed using the symplectic integrator [26].

Note that if the condition $p_{\mathrm{m}}<b$ holds, there are two global equilibria of (2) at $\left(\delta_{i}, \omega_{i}\right)=\left(\delta_{\mathrm{c}}=\sin ^{-1}\left(p_{\mathrm{m}} / b\right), 0\right)$, which is a global energy minimum of elliptic type, and $\left(\pi-\delta_{\mathrm{c}}, 0\right)$ of the hyperbolic type. Let us consider a local disturbance given by the following set of initial conditions, close to the elliptic equilibrium:

$$
\left(\delta_{i}(0), \omega_{i}(0)\right)= \begin{cases}(-0.352,0) & \text { for } i=N / 2, \\ \left(\delta_{\mathrm{c}}, 0\right) & \text { else. }\end{cases}
$$

Physically, generator $N / 2$ is locally disturbed, while the other generators are at their steady states, that is, the elliptic equilibrium. Fig. 2 shows sequential snapshots of angular positions $\delta_{i}$ for coupled swing dynamics caused by the local disturbance. The red points on the circle denote the angular positions of $N$ generators. At the initial time in Fig. 2(a), generator $N / 2$ is disturbed, and its initial position $\delta_{N / 2}(0)$ is denoted by the lower red point, while all the other generators are at the same position on the circle, denoted by the upper red point. In the snapshots shown in Figs. 2(b)-(j), the angular positions of $N$ generators are in an intermediate regime of linear oscillations around their steady states (these snapshots can be compared to Fig. 3(a) where the average angle vs. time is plotted). These oscillations are bounded and essentially linear. However, in Figs. 2(j)-(n), they start to show coherent growth. Finally, they grow unbounded in Figs. 2(n)-(t). The unbounded growth indicates that all the generators lose synchronism with the

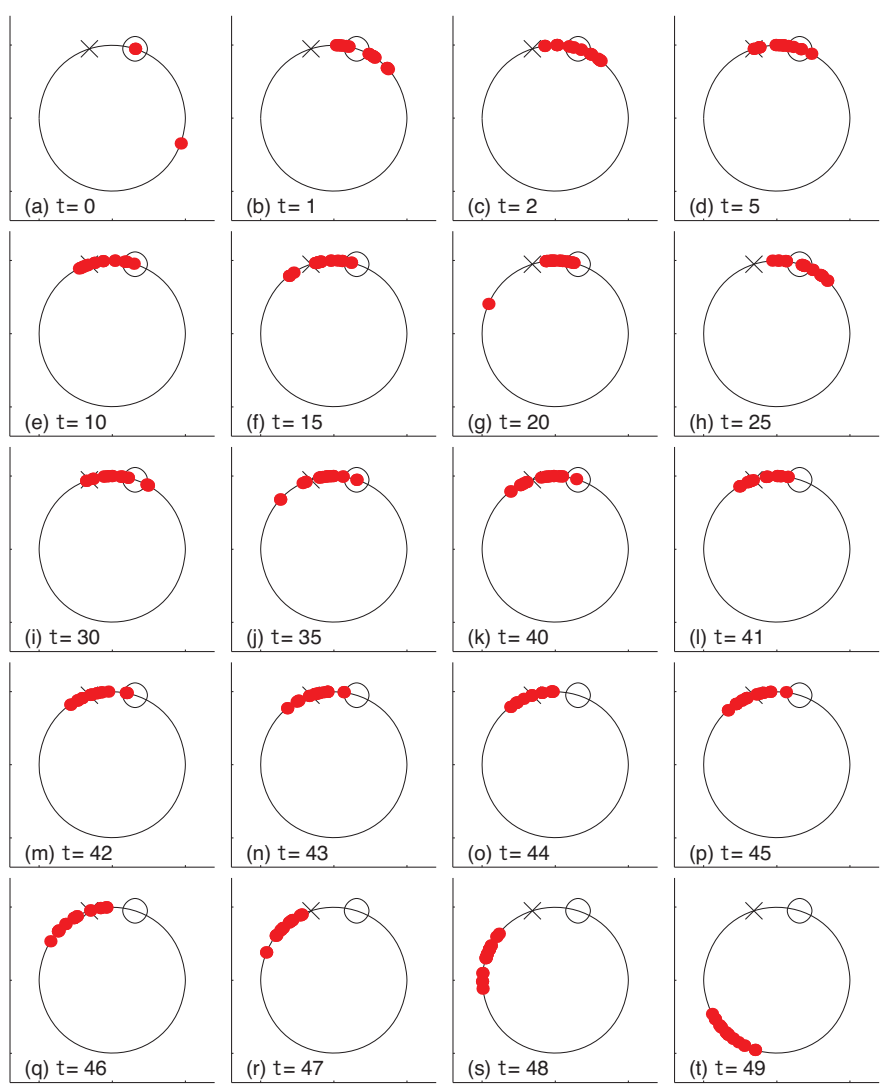

Fig. 2. Sequential snapshots of angular positions $\delta_{i}(t)$ for coupled swing dynamics in the loop power system. Red points on the circle denote the angular positions $\delta_{i} \in \mathbb{T}^{1}$ of $N$ generators and move according to (2) from the initial condition (4). At the initial time (a), generator $N / 2$ is disturbed, and its initial position $\delta_{N / 2}(0)$ is denoted by the lower red point, while all the other generators are at the elliptic equilibrium $\delta_{i}=\delta_{\mathrm{c}}$, denoted by the circle $\circ$. The symbol $\times$ on the circle denotes the position of the hyperbolic equilibrium $\delta_{i}=\pi-\delta_{\mathrm{c}}$.

infinite bus. This is the phenomenon that we call the Coherent Swing Instability (CSI). It represents a dynamic transition from a localized perturbation that initially causes linear multi-swing (or inter-machine mode) oscillations and ultimately leads to coherent loss of synchronism of the whole group of generators with the infinite bus, that is, instability of inter-area mode oscillation.

Note that in Fig. 2 we plot $t$ in units of $\sqrt{2 H / \omega_{\mathrm{b}}}$ where $H$ is the per-unit time constant of each machine, and $\omega_{\mathrm{b}}$ the system angular frequency. For $H=10 \mathrm{~s}$ and $\omega_{\mathrm{b}}=2 \pi \times(60 \mathrm{~Hz})$, $t=50$ is equal to $12 \mathrm{~s}$ in real time. Hence the onset time of instability in Fig. 2 is within the short-term regime. Thus we have discussed the terms that lead to linear oscillations and loss of coherence at the end of the previous paragraph, and connected them to descriptions of various types of instabilities described in power systems literature.

\section{The Mechanism}

In this section, we summarize the dynamical mechanism of CSI for the loop power system. After introducing collective dynamics, we discuss the CSI in terms of escape phenomena 
[22], [23] and dynamical systems close to internal resonance [24].

1) Collective Dynamics and Escape Phenomenon: The collective-phase variable $\delta$ and its time derivative $\omega$ for the loop power system are defined as

$$
\delta=\frac{1}{N} \sum_{i=1}^{N} \delta_{i}, \quad \omega=\frac{\mathrm{d} \delta}{\mathrm{d} t}=\frac{1}{N} \sum_{i=1}^{N} \omega_{i} .
$$

The variables are well known in power system stability analysis as the COA (Center-Of-Angle) [19] or COI (Center-OfInertia) [1]. Time evolution of these variables, called collective dynamics, of the loop system is given by

$$
\frac{\mathrm{d} \delta}{\mathrm{d} t}=\omega, \quad \frac{\mathrm{d} \omega}{\mathrm{d} t}=p_{\mathrm{m}}-\frac{b}{N} \sum_{i=1}^{N} \sin \delta_{i} .
$$

Fig. 3 shows examples of time evolution of collective-phase variable $\delta$ and trajectory on $\delta-\omega$ plane. The red line is for the initial condition $\delta_{N / 2}(0)=-0.352$ and corresponds to the CSI shown in Fig. 2. The blue line is for $\delta_{N / 2}(0)=0$ and does show to any instability. The two boxes (ם) denote the equilibria of the following system:

$$
\frac{\mathrm{d} \delta}{\mathrm{d} t}=\omega, \quad \frac{\mathrm{d} \omega}{\mathrm{d} t}=p_{\mathrm{m}}-b \sin \delta .
$$

That is, the system represents local dynamics of single generator. It also describes dynamics on the two-dimensional invariant manifold $\mathcal{I}$. The variables $(\delta, \omega)$ are regarded as the local coordinates of this manifold. The system (5) with $\left|p_{\mathrm{m}} / b\right|<1$ has the saddle point $\left(\pi-\delta_{\mathrm{c}}, 0\right)$ connected to itself by the homoclinic orbit $\Gamma_{0}$ as shown in Fig. 3(b).

Now we compare dynamics of the full system (2) projected onto $\delta-\omega$ plane with the dynamics of the system (5). For the red trajectory showing the divergence in Fig. 3(b), the intermediate linear oscillations in Figs. 2(b)-(j) appear as a trapped motion inside $\Gamma_{0}$, and the final coherent growth in Figs. 2(j)-(q) appears as a divergence motion over $\Gamma_{0}$. Trajectories of (5) are bounded oscillations (librations) inside $\Gamma_{0}$ and never diverge, or always diverge outside of it. Therefore, the homoclinic orbit $\Gamma_{0}$ defines a boundary of stable region, and we say that CSI occurs when a trajectory projected onto $\delta-\omega$ plane starts inside the region defined by $\Gamma_{0}$, escapes it, and diverges to infinity.

2) Escape Phenomenon and Resonance: We investigate the escape phenomenon with dynamical systems theory close to resonance. To do so, by using the normal mode and actionangle variable transformations, we re-write (2) as

$$
\begin{aligned}
\frac{\mathrm{d} I_{n}}{\mathrm{~d} t} & =\epsilon F_{n}\left(I_{1}, \ldots, I_{N-1}, \delta, \theta_{1}, \ldots, \theta_{N-1}\right), \\
\frac{\mathrm{d} \theta_{n}}{\mathrm{~d} t} & =\Omega_{n}+\epsilon G_{n}\left(I_{1}, \ldots, I_{N-1}, \delta, \theta_{1}, \ldots, \theta_{N-1}\right), \\
\frac{\mathrm{d} \omega}{\mathrm{d} t} & =\epsilon\left(\tilde{p}_{\mathrm{m}}-\frac{\tilde{b}}{N} \sum_{i=1}^{N} \sin \delta_{i}\left(I_{1}, \ldots, I_{N-1},\right.\right. \\
\frac{\mathrm{d} \delta}{\mathrm{d} t} & \left.\left.=\omega . \theta_{1}, \ldots, \theta_{N-1}\right)\right),
\end{aligned}
$$
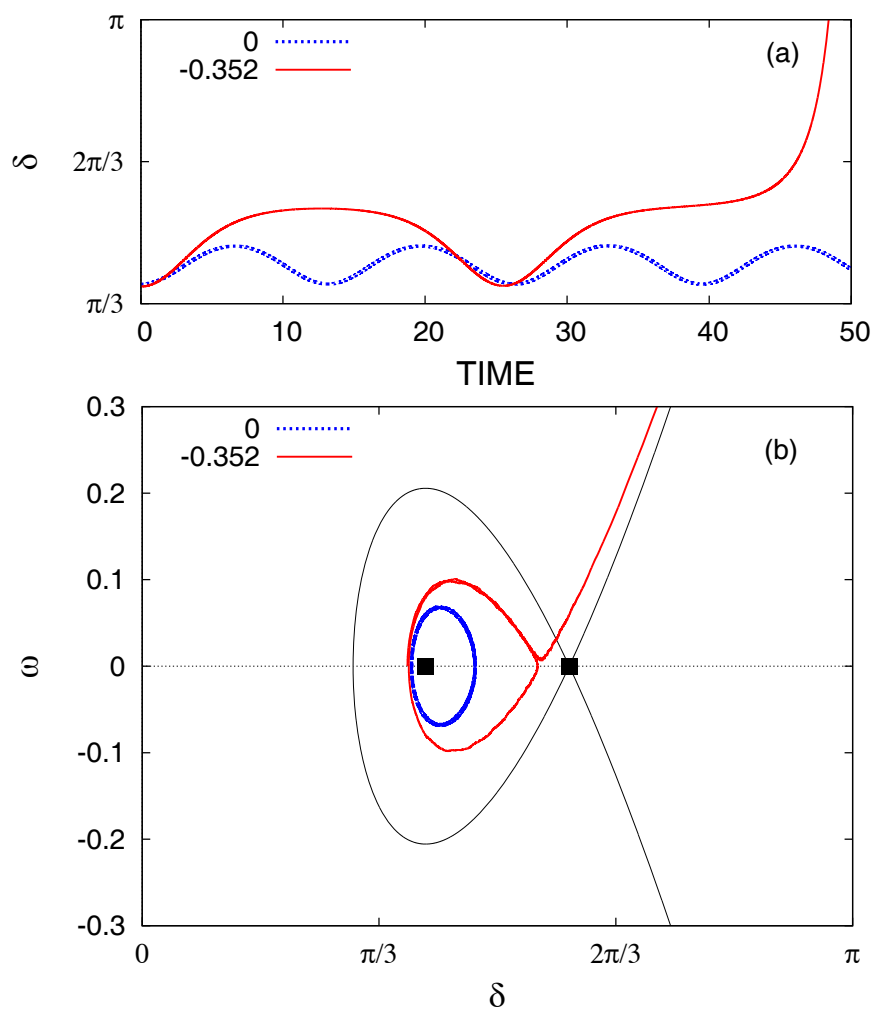

Fig. 3. Collective dynamics of coupled swings in the loop power system for the local disturbances $\delta_{N / 2}(0)=0$ and -0.352 : (a) $t-\delta$ curves and (b) trajectories on $\delta-\omega$ plane

The derivation of (6) is presented in [11]. The integer $n \in$ $\{1,2, \ldots, N-1\}$ stands for the label of $N-1$ linear oscillatory modes under $\epsilon=0$. The variable $I_{n} \in \mathbb{R}^{+}$denotes the action variable of the $n$-th linear mode, and $\theta_{n} \in \mathbb{T}^{1}$ its angle variable. The angular frequency of the $n$-th mode is given by $\Omega_{n}=2 \sqrt{\left|b_{\text {int }}\right|}|\sin (\pi n / N)|$. The constant $\epsilon$ stands for the perturbation parameter satisfying $p_{\mathrm{m}}=\epsilon \tilde{p}_{\mathrm{m}}$ and $b=\epsilon \tilde{b}$, and we choose its value such that the orders of magnitudes of parameters $\Omega_{n}, \tilde{p}_{\mathrm{m}}, \tilde{b}$, and of functions $F_{n}$ and $G_{n}$ are the same.

Consider the system (6) from a viewpoint of slow-fast dynamics. Since the values of parameters $p_{\mathrm{m}}$ and $b$ are smaller than $b_{\text {int }}$, the perturbation parameter $\epsilon$ becomes small, and the frequency $\Omega_{n}$ becomes larger than the other parameters. This clearly indicates that the CSI phenomenon occurs in the dynamical system with one nonlinear mode that is weak relative to linear oscillatory modes. Here the variables $I_{n}$ and $\omega$ are slow due to the presence of $\varepsilon$, and the variables $\theta_{n}$ are fast. Here the remaining variable $\delta$ becomes semi-fast because it changes fast except for the neighborhood of $\delta$-axis, namely, the set $\left\{\left(I_{1}, \ldots, I_{N-1}, \omega, \theta_{1}, \ldots, \theta_{N-1}, \delta\right) \in\left(\mathbb{R}^{+}\right)^{N-1} \times\right.$ $\left.\mathbb{R} \times \mathbb{T}^{N}=: \mathbb{X} \mid \omega=0\right\}$. The set becomes the surface for resonance of all orders ${ }^{2}$ in (6). The so-called resonant surface

${ }^{2}$ For $\boldsymbol{k}=\left(0,0, \ldots, 0, k_{N}\right)^{\mathrm{T}}$ (for all $k_{N} \in \mathbb{Z} \backslash\{0\}$ ) and $\boldsymbol{\Omega}(\omega)=$ $\left(\Omega_{1}, \ldots, \Omega_{N-1}, \omega\right)^{\mathrm{T}}$, we have the resonance condition $\boldsymbol{k}^{\mathrm{T}} \boldsymbol{\Omega}(\omega)=k_{N} \omega=$ 


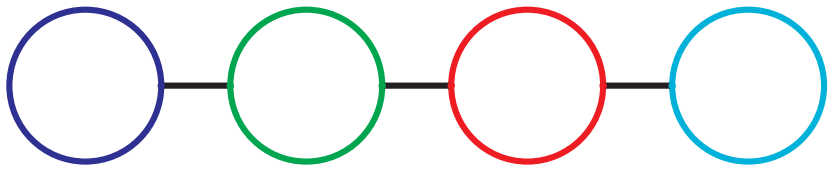

Fig. 4. A network of weakly interconnected loop power systems. The system consists of the $N_{\mathrm{s}}=4$ loop power systems and weak transmission lines joining them.

is a $(2 N-1)$-dimensional (codimension one) subspace in the full phase space $\mathbb{X}$. Now recall the red trajectory in Fig. 3 which describes the CSI. The trajectory starts on the resonant surface and escapes from the region defined by $\Gamma_{0}$. Thus we say that the CSI occurs when a trajectory projected onto $\delta-\omega$ plane escapes from the resonant surface. Whether the escape happens or not depends on initial conditions of (6). The region of stability in which any trajectory does not escape is estimated analytically [9], [11] and numerically [13].

\section{Coupled SWing Dynamics Leading to CASCADING FAILURE}

In this section, we study a phenomenon of short-term swing dynamics leading to cascading failure in a network of weakly interconnected loop systems. The system consists of multiple loop power systems that are weakly coupled (Sec. III-A). After introducing the swing equations (Sec. III-B), we present coupled swing dynamics of the network and show that the CSI happens for all of the loop systems in a cascade manner (Sec. III-C). We analyze the cascading failure by projecting the full-system dynamics onto the phase planes of COA variables and describe simple dynamics underlying in it (Sec. III-D). This section uses a relatively simple model of power systems to analyze cascading failures. Detailed analysis using a realistic model of power systems is submitted to an archival journal [11].

\section{A. A Model of Power Network}

Consider the network of weakly interconnected power systems shown in Fig. 4. The system includes the $N_{\mathrm{s}}$ loop systems and weak interconnections. We call each loop system as the sub-system and suppose that each sub-system has the equal specification of $N$ synchronous generators and network topology. The number $N$ is supposed to be odd. The $N_{\mathrm{s}}$ sub-systems are joined to each other in series via weak interconnections. The bus on generator $N / 2+1$ in sub-system $\# i\left(i=1, \ldots, N_{\mathrm{s}}-1\right)$ and the bus on generator 1 in subsystem \# $(i+1)$ are joined by a transmission line. For modeling and analysis, we make the additional two assumptions. (vi) The infinite bus is outside of the network, and it weakly and equally interacts with every sub-system. (vii) The magnitudes of interconnections between two sub-systems are the same and are weak compared with those between generators within a sub-system. Thus, we weakly interconnect the $N_{\mathrm{s}}$ sub-systems, in each of which the $N$ synchronous machines are strongly connected.

\section{B. The Swing Equations}

We use the swing equations for analysis of coupled swing dynamics. Under the assumptions (i)-(vii) the short-term swing dynamics of generator $j$ in sub-system $\# i(j=1, \ldots, N$ and $i=1, \ldots, N_{\mathrm{s}}$ ) are represented by

$$
\left.\begin{array}{rl}
\frac{\mathrm{d} \delta_{i j}}{\mathrm{~d} t}= & \omega_{i j}, \\
\frac{\mathrm{d} \omega_{i j}}{\mathrm{~d} t}= & p_{\mathrm{m}}-b \sin \delta_{i j}-b_{\mathrm{int}}\left\{\sin \left(\delta_{i j}-\delta_{i, j-1}\right)\right. \\
& \left.+\sin \left(\delta_{i j}-\delta_{i, j+1}\right)\right\} \\
& -\epsilon b_{\mathrm{int}} g_{i j}\left(\delta_{i-1, N / 2+1}, \delta_{i j}, \delta_{i+1, N / 2+1}\right),
\end{array}\right\}
$$

where $g_{i j}$ represents the coupling between sub-systems, given by

$g_{i j}= \begin{cases}\sin \left(\delta_{i j}-\delta_{i-1, N / 2+1}\right) & \text { for } i \neq 1, j=1, \\ \sin \left(\delta_{i j}-\delta_{i+1, N / 2+1}\right) & \text { for } i \neq N_{\mathrm{s}}, j=N / 2+1, \\ 0 & \text { else. }\end{cases}$

The loop topology for each sub-system induces the following conditions:

$$
\delta_{i, 0}(t)=\delta_{i, N}(t), \quad \delta_{i, N+1}(t)=\delta_{i, 1}(t),
$$

where $i=1, \ldots, N_{\mathrm{s}}$. The variable $\delta_{i j}$ is the angular position of rotor in generator $j$ in sub-system $\# i$ with respect to the infinite bus. The variable $\omega_{i j}$ is the non-dimensional deviation of rotor speed in generator $i$ relative to system angular frequency. The parameters $p_{\mathrm{m}}, b, b_{\text {int }}$, and $N$ are the same as in the single loop system. The parameter $\epsilon$ controls the magnitude of coupling strength between sub-systems. The setting $\epsilon=0$ implies that there is no coupling between sub-systems. We will fix $\epsilon$ at a small value to explore the network of weak inter-system connection and strong inter-machine one.

Now we simplify (7) in the same way as the single loop system. Under the sufficiently small $\epsilon$, in each sub-system the differences between individual angular positions stay small for all time. Thus we investigate the dynamics of each subsystem close to the invariant manifold $\mathcal{I}$. This allows us to use the first-order approximation of the sinusoidal coupling term $b_{\text {int }}\left\{\sin \left(\delta_{i j}-\delta_{i, j-1}\right)+\sin \left(\delta_{i j}-\delta_{i, j+1}\right)\right\}$ and to derive the following system:

$$
\left.\begin{array}{rl}
\frac{\mathrm{d} \delta_{i j}}{\mathrm{~d} t}= & \omega_{i j}, \\
\frac{\mathrm{d} \omega_{i j}}{\mathrm{~d} t}= & p_{\mathrm{m}}-b \sin \delta_{i j}-b_{\mathrm{int}}\left\{\left(\delta_{i j}-\delta_{i, j-1}\right)\right. \\
& \left.+\left(\delta_{i j}-\delta_{i, j+1}\right)\right\} \\
& -\epsilon b_{\mathrm{int}} g_{i j}\left(\delta_{i-1, N / 2+1}, \delta_{i j}, \delta_{i+1, N / 2+1}\right) .
\end{array}\right\}
$$

The system (8) has the nonlinearity in the local term and the coupling term between sub-systems. We adopt (8) to analyze short-term swing dynamics of the network in Fig. 4.

\section{A Phenomenology}

We numerically simulate coupled swing dynamics of the $N \times N_{\mathrm{s}}$ generators. The parameter settings of $p_{\mathrm{m}}, b$, and $b_{\text {int }}$ are the same as (3), and the parameters $\epsilon$ and $N_{\mathrm{s}}$ are set as follows:

$$
\epsilon=0.1, \quad N_{\mathrm{s}}=4 .
$$



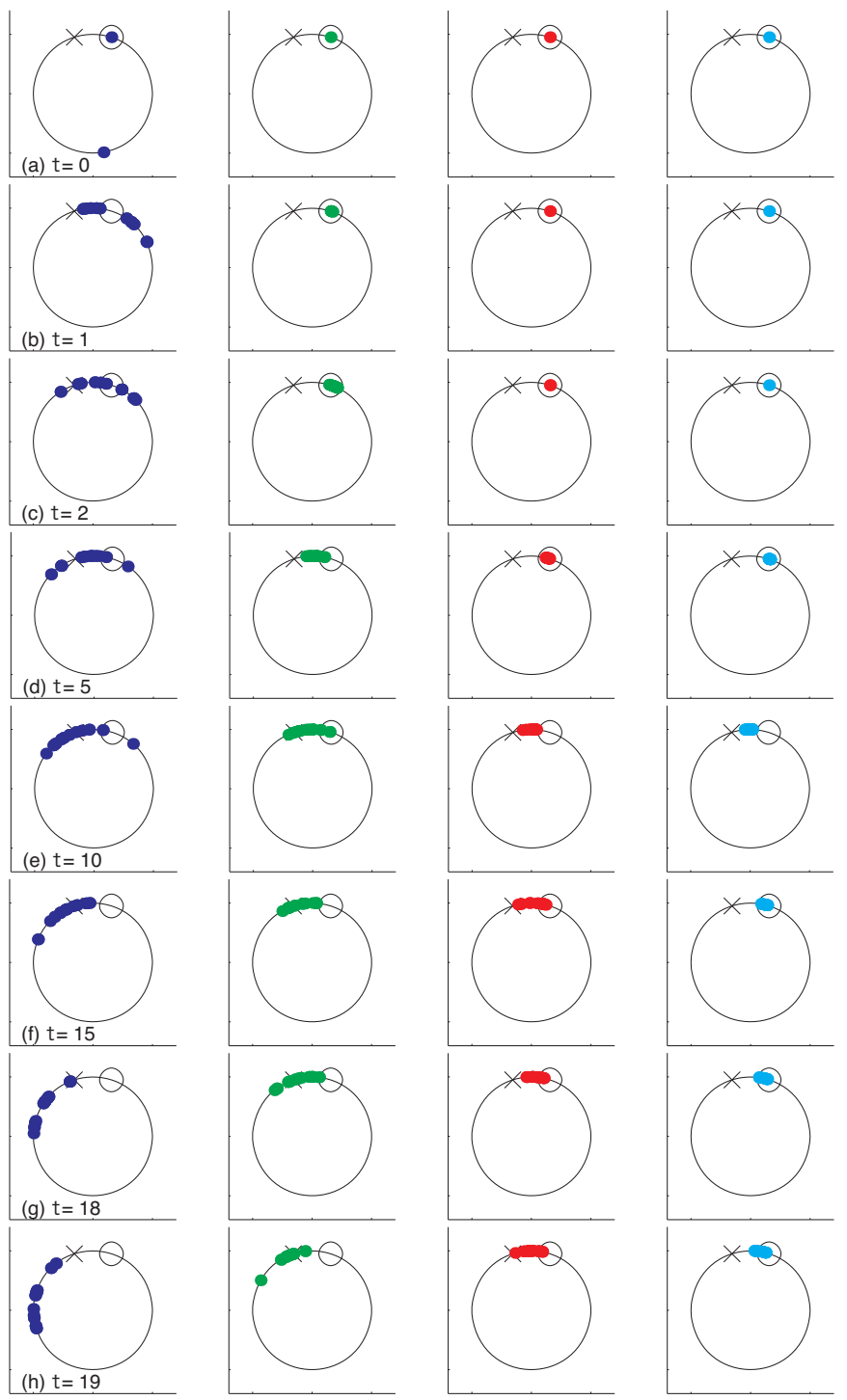

Fig. 5. Sequential snapshots of angular positions $\delta_{i j}(t)$ for coupled swing dynamics in the network of weakly interconnected loop power systems. At the initial time (a), generator $N / 2$ at sub-system \#1 is disturbed, and its initial position $\delta_{N / 2}(0)$ is denoted by the lower blue point, while the other generators are at the elliptic equilibrium $\delta_{i}=\delta_{\mathrm{c}}$, denoted by the circle $\circ$. The symbol $\times$ on the circle denotes the position of the hyperbolic equilibrium $\delta_{i}=\pi-\delta_{\mathrm{c}}$.

In this setting, the magnitude of interaction between machines is larger than that between sub-systems. The magnitude of interaction between sub-systems is larger than that of a generator with the infinite bus, characterized by $p_{\mathrm{m}}$ and $b$. Note that if the condition $p_{\mathrm{m}}<b$ holds, there are two global equilibria of $(8)$ at $\left(\delta_{i}, \omega_{i}\right)=\left(\delta_{\mathrm{c}}, 0\right)$, which is a global energy minimum of elliptic type, and $\left(\pi-\delta_{\mathrm{c}}, 0\right)$. In the following, we consider a local disturbance given by the following set of initial conditions, close to the elliptic equilibrium:

$$
\left(\delta_{i j}(0), \omega_{i j}(0)\right)= \begin{cases}(-1.39,0) & \text { for } i=1, j=1 \\ \left(\delta_{\mathrm{c}}, 0\right) & \text { else. }\end{cases}
$$
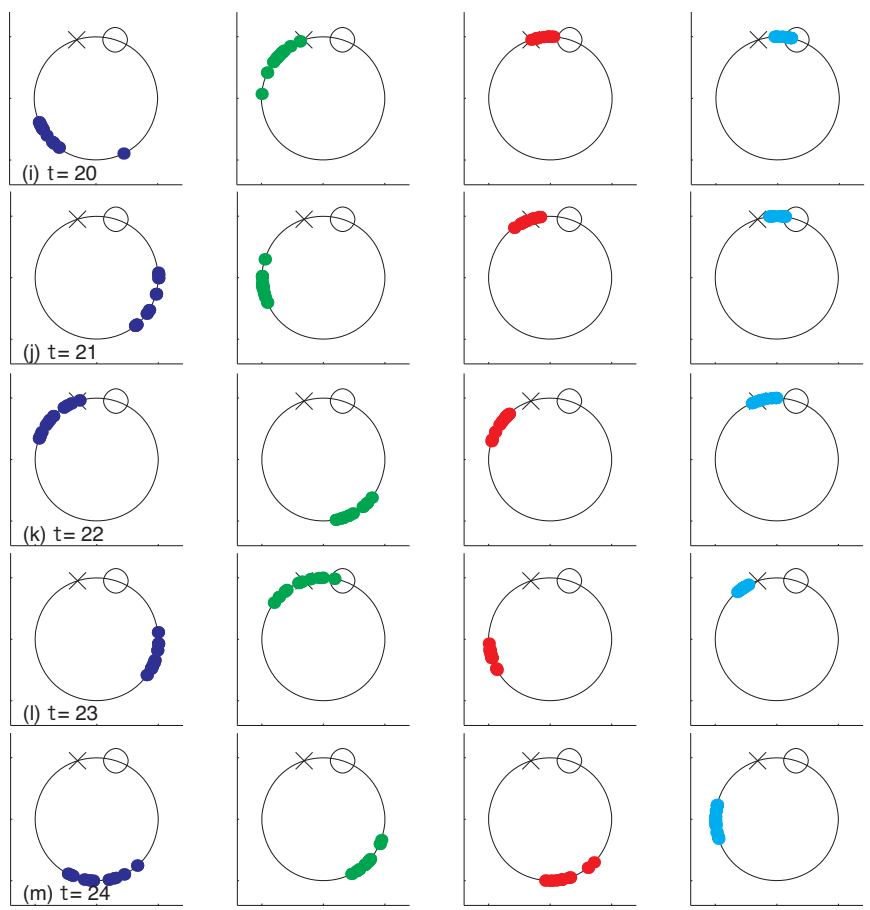

Fig. 5. ( continued)

This initial condition physically implies a local disturbance at generator 1 in sub-system \#1. Fig. 5 shows sequential snapshots of angular positions $\delta_{i j}$ for coupled swing dynamics caused by the local disturbance. The blue points on the circle denote the angular positions of $N$ generators in sub-system $\# 1$, the green points for sub-system \#2, the red points for sub-system \#3, and the cyan points for sub-system \#4. The local disturbance happens in sub-system \#1. At the initial time in Fig.5(a), generator 1 is disturbed, and its initial position $\delta_{1,1}(0)$ is denoted by the lower blue point, while the other generators are at the same position on the circle, denoted by the upper points. In the snapshots of Figs. 5(b)(e), the angular positions of generators are in an intermediate regime of bounded swings (these snapshots can be compared to Fig. 6(a) where the average angle vs. time is plotted). In this regime, the swings for sub-systems \#2-\#4 become smaller than those for sub-system \#1, because of the weakness of interconnections. In Figs. 5(f)-(h) the angular positions for sub-system \#1, denoted by the blue points, grow coherently. Finally, they grow unbounded in Figs. 5(i)-(m). After this, following the coherent growth in sub-system \#1, the angular positions for sub-system \#2, denoted by the green points, grow coherently in Figs. 5(g)-(j) and finally grow unboundedly in Figs. $5(\mathrm{k})-(\mathrm{m})$. In the same way, initiated by the cascade, the angular positions for sub-system \#3, denoted by the red points, grow coherently in Figs. 5(j)-(m), and the angular positions for sub-system \#4, denoted by the cyan points, do in Figs. 5(k)(m). In this way, the cascade of coherent divergences continues up to the last sub-system. The coherent divergence of each subsystem corresponds to its transient instability and hence to a 
sub-system failure. Fig. 5 demonstrates that one local and finite disturbance causes coupled swing dynamics in the network and initiates the cascade of sub-system failures.

\section{The Mechanism}

In the single loop system, we were able to capture the motion of a hidden nonlinear mode by projecting the fullsystem dynamics onto the phase plane of COA variables. We investigate the cascade of sub-system failures using the notion of COA. Here it is not effective to define the COA for the full-system dynamics of $N \times N_{\mathrm{s}}$ generators, because it does not provide any insight of the interaction of different subsystems. In this case, we define the COA for each sub-system $i\left(i=1, \ldots, N_{\mathrm{s}}\right)$ as

$$
\delta^{i}=\frac{1}{N} \sum_{j=1}^{N} \delta_{i j}, \quad \omega^{i}=\frac{1}{N} \sum_{j=1}^{N} \omega_{i j},
$$

where $\delta^{i}$ is the COA of sub-system $\# i$, and $\omega^{i}$ its time derivative. The variables describe the averaged motion of all the generators in sub-system \#i. Fig. 6 plots (a) the time evolution of COA $\delta^{i}$ and (b) the trajectories of (8) on $\delta^{i}$ $\omega^{i}$ planes which show the cascading dynamics in Fig. 5. In all the sub-systems, the trajectories move in a similar way to the escape phenomenon observed in Fig. 3(b). In sub-system \#1, the trajectory remains in the vicinity of $\delta^{1}$-axis for a while and begins to diverge to the infinite. In sub-system \#2, the trajectory is kicked by the divergence of sub-system \#1 and finally diverges. We can see similar behaviors of trajectories for sub-systems \#3 and \#4.

In order to investigate the collective dynamics in Fig. 6, we present equations for the COA motions. The differential equations of $\delta^{i}$ and $\omega^{i}\left(i=1, \ldots, N_{\mathrm{s}}\right)$ are derived in the same way as in (5):

$$
\left.\begin{array}{rl}
\frac{\mathrm{d} \delta^{i}}{\mathrm{~d} t}= & \omega^{i}, \\
\frac{\mathrm{d} \omega^{i}}{\mathrm{~d} t}= & p_{\mathrm{m}}-\frac{b}{N} \sum_{j=1}^{N} \sin \delta_{i j} \\
& -\epsilon \frac{b_{\text {int }}}{N} g_{i}\left(\delta_{i-1, N / 2+1}, \delta_{i, 1}, \delta_{i, N / 2+1}, \delta_{i+1,1}\right),
\end{array}\right\}
$$

where $g_{i}$ contains the interconnection terms $g_{i j}$, and the magnitude is of order 1. In the current settings of parameters, all the terms on the right-hand side of the differential equation of $\omega^{i}$ are of order 1 at most. The equations are close to (5) and suggest that the COA motions of every sub-system can be discussed with the analogy to single degree-of-freedom swing equations with external excitations.

These observations give a dynamical mechanism of the cascading failure in Fig. 5. In all of the sub-systems, the dynamics are mainly governed by the dynamical system equivalent to the loop power system in Sec. II and are perturbed by weak external perturbations (of order $\varepsilon b_{\text {int }} / N$ ) that originate from the interaction with other sub-systems. Recall that in the loop system, CSI occurs when a trajectory escapes from the vicinity of the resonant surface corresponding to the set (a)
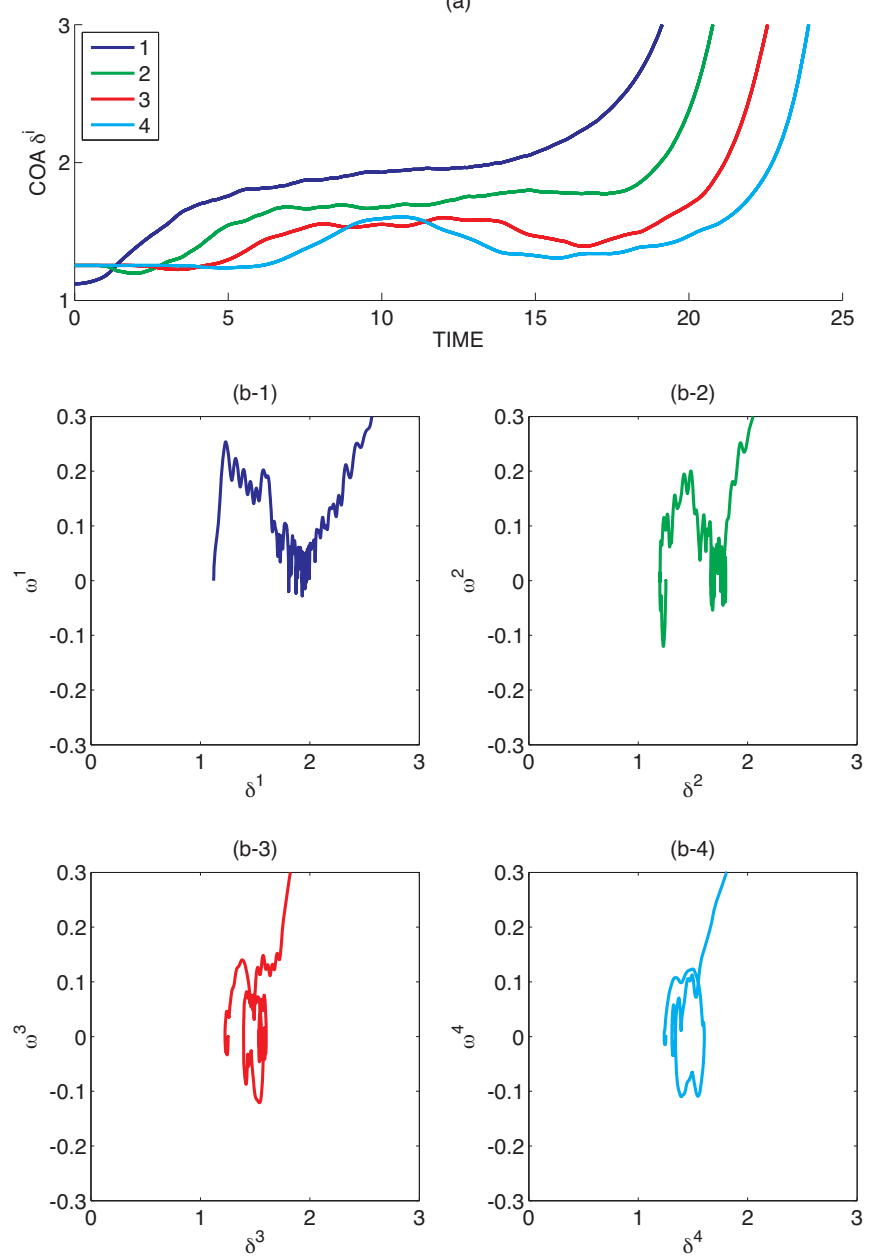

Fig. 6. Collective dynamics of coupled swings in the network of weakly interconnected loop power systems: (a) $t-\delta^{i}$ curves and (b) trajectories on $\delta^{i}-\omega^{i}$ planes. These plots correspond to the dynamics of Center-Of-Angle (COA) variables (9).

$\{\omega=0\}$, namely, $\delta$-axis. In sub-system \#1, the trajectory starts on $\delta^{1}$-axis and finally escapes it. This escape is initiated by an external perturbation of interaction with sub-system \#2. Thus we can say that the divergent motion of COA in subsystem \#1 is the escape from resonance due to small and temporal external perturbations. The dynamics of the other sub-systems are essentially explained using a similar argument of resonance. The sequence of escape phenomena results from the interactions between sub-systems. Thus we can say that the cascading failure in Fig. 5 occurs due to the sequence of escape phenomena of the sub-systems consisting of the power network.

\section{SUMmARY AND Discussion}

In this paper, we reported short-term swing dynamics leading to cascading failure in an interconnected power system. The dynamics were observed for the network of multiple loop power systems shown in Fig. 4. We numerically show the cascade of Coherent Swing Instabilities (CSIs), which 
have been studied in [9]-[13], in the power network. The contribution of this paper is to give the dynamical mechanism of cascading failure. The mechanism is the sequence of escape phenomena in collective (center-of-angle or slow-coherent) modes for every loop power system.

Cascading failures are fairly complicated phenomena emerging in complex dynamical systems. It would be impossible to obtain a simple mechanism that can explain all dynamics and events in cascading failures. For a power grid consisting of many sub-grids, the instability reviewed in Sec. II describes a failure of one sub-grid caused by transient instability. In Sec. III we show that a sequence of sub-grid failures is induced by a sequence of CSIs. Thus we suggest that CSI could be a part of the dynamical mechanism of cascading failures in power grids. The analysis which we performed here uses the relatively simple model. Detailed analysis of the mechanism using a realistic model of power systems is in an archival journal paper [11].

It is often said that cascading failures happen since voltage or frequency fluctuations cause unnecessary relay actions. This is naturally modeled by a hybrid dynamical system. In [27] the authors indeed use a hybrid model to analyze swing dynamics leading to the cascading failure in the September 2003 blackout in Italy. Analysis of the hybrid model shows that the swing dynamics, especially desynchronization of individual generators, result from a network switching with a simple relay feedback control. In this paper, on the other hand, we describe another scenario of swing dynamics leading to desynchronization of individual generators without any network switching. This is a counterexample to the standard argument as stated in the beginning of this paragraph. Of course, the dynamic phenomenon that we studied in this paper may be an extreme example for cascading failures. Our mechanism of cascading failures will need further research in real data on cascading failures.

\section{ACKNOWLEDGMENTS}

This research is supported in part by the JSPS Postdoctoral Fellowships for Research Abroad and the Grant-in-Aid for the Global COE Program 'Education and Research on Photonics and Electronics Science and Engineering" from the MEXT of Japan.

\section{REFERENCES}

[1] P. Kundur, Power System Stability and Control. McGraw-Hill, 1994.

[2] H. D. Chiang, "Power system stability," in Wiley Encyclopedia of Electrical and Electronics Engineering, J. G. Webster, Ed. New York: John Wiley \& Sons, March 1999, pp. 105-137.

[3] S. Corsi and C. Sabelli, "General blackout in Italy Sunday September 28, 2003, h. 03:28:00," in Proceedings of the IEEE PES General Meeting, vol. 2, Denver, USA, June 2004, pp. 1691-1702.

[4] N. Kopell and R. B. Washburn Jr., "Chaotic motions in the twodegree-of-freedom swing equations," IEEE Transactions on Circuits and Systems, vol. CAS-29, no. 11, pp. 738-746, November 1982.

[5] E. H. Abed and P. P. Varaiya, "Nonlinear oscillations in power systems," Electrical Power \& Energy Systems, vol. 6, no. 1, pp. 37-43, January 1984.

[6] F. M. A. Salam, J. E. Marsden, and P. P. Varaiya, "Arnold diffusion in the swing equations of a power system," IEEE Transactions on Circuits and Systems, vol. CAS-31, no. 8, pp. 673-688, August 1984.
[7] M. Varghese and J. S. Thorp, "An analysis of truncated fractal growths in the stability boundaries of three-node swing equation," IEEE Transactions on Circuits and Systems, vol. 35, no. 7, pp. 825-834, July 1988.

[8] Y. Ueda, T. Enomoto, and H. B. Stewart, "Chaotic transients and fracta structures governing coupled swing dynamics," in Applied Chaos, J. H. Kim and J. Stringer, Eds. London: John Wiley \& Sons, 1992, ch. 8.

[9] Y. Susuki, I. Mezić, and T. Hikihara, "Global swing instability of multimachine power systems," in Proceedings of the 47th IEEE Conference on Decision and Control, Cancun, Mexico, December 9-11 2008, pp. 2487-2492.

[10] — "Global swing instability in the New England power grid model," in Proceedings of the 2009 American Control Conference, St. Luis, United States, June 10-12 2009, pp. 3446-3451.

[11] _ _ "Coherent swing instability of power grids," (submitted to Journal of Nonlinear Science).

[12] - "Coherent dynamics and instability of power grids," in Proceedings of the 2009 International Symposium on Nonlinear Theory and its Applications, Sapporo, Japan, October 18-21 2009, pp. 540-543.

[13] Y. Susuki and I. Mezić, "Ergodic partition of phase space in continuous dynamical systems," in Proceedings of the 48th IEEE Conference on Decision and Control, Shanghai, China, December 16-18 2009, (to appear).

[14] I. Mezić, "Dynamics and control of large-scale molecular motion," in Preprint of IFAC Symposium.

[15] - "On the dynamics of molecular conformation," Proceedings of the National Academy of Sciences of the USA, vol. 103, no. 20, pp. 7542-7547, 2006

[16] B. Eisenhower and I. Mezić, "A mechanism for energy transfer leading to conformation change in networked nonlinear systems," in Proceedings of the 46th IEEE Conference on Decision and Control, New Orleans, USA, December 2007, pp. 3976-3981.

[17] P. Du Toit, I. Mezić, and J. Marsden, "Coupled oscillator models with no scale separation," Physica D, vol. 238, no. 5, pp. 490-501, March 2009.

[18] S. Wiggins, Global Bifurcations and Chaos: Analytical Methods, ser. Applied Mathematical Sciences. New York: Springer-Verlag, 1988, vol. 73.

[19] T. Athay, R. Podmore, and S. Virmani, "A practical method for the direct analysis of transient stability," IEEE Transactions on Power Appratatus and Systems, vol. PAS-98, no. 2, pp. 573-584, March/April 1979.

[20] G. Peponides, P. V. Kokotović, and J. H. Chow, "Singular perturbations and time scales in nonlinear models of power systems," IEEE Transactions on Circuits and Systems, vol. CAS-29, no. 11, pp. 758-766, November 1982.

[21] Y. Susuki and I. Mezić, "Nonlinear Koopman modes of coupled swing dynamics and coherency identification," in 2010 IEEE Power \& Energy Society General Meeting, Minneapolis, United States, July 25-29 2010, (submitted)

[22] J. M. T. Thompson and H. B. Stewart, Nonlinear Dynamics and Chaos, 2nd ed. England: John Wiley \& Sons, 2002.

[23] S. Wiggins, Chaotic Transport in Dynamical Systems, ser. Interdisciplinary Applied Mathematics. New York: Springer-Verlag, 1992, vol. 2.

[24] V. I. Arnold, V. V. Kozlov, and A. I. Neishtadt, Mathematical Aspects of Classical and Celestial Mechanics, 3rd ed., ser. Encyclopaedia of Mathematical Sciences. Heidelberg Germany: Springer-Verlag, 2006, vol. 3, chapter 6 .

[25] IEEE PES CAMS Task Force on Cascading Failure, Initial review of methods for cascading failure analysis in electric power transmission, in Proceedings of the IEEE PES General Meeting, Pittsburgh, USA, July 2008, pp. 1-8.

[26] H. Yoshida, "Construction of higher order symplectic integrators," Physics Letters A, vol. 150, no. 5-7, pp. 262-268, November 121990.

[27] Y. Susuki, Y. Takatsuji, and T. Hikihara, "Hybrid model for cascading outage in a power system: A numerical study," IEICE Transactions on Fundamentals of Electronics, Communications and Computer Sciences, vol. E92-A, no. 3, pp. 871-879, March 2009. 